\title{
Article \\ Research on Intellectualized Location of Coal Gangue Logistics Nodes Based on Particle Swarm Optimization and Quasi-Newton Algorithm
}

\author{
Shengli Yang ${ }^{1}$, Junjie Wang ${ }^{2, *}$, Ming $\mathrm{Li}^{1, *}$ and Hao Yue ${ }^{1}$ \\ 1 School of Energy and Mining Engineering, China University of Mining and Technology (Beijing), \\ Beijing 100083, China; 108992@cumtb.edu.cn (S.Y.); bqt2100101020@student.cumtb.edu.cn (H.Y.) \\ 2 China National Coal Group Corporation, Beijing 100120, China \\ * Correspondence: wangjunjie5@chinacoal.com (J.W.); zqt1900101015g@student.cumtb.edu.cn (M.L.); \\ Tel.: +86-13051178588 (J.W.); +86-17614131040 (M.L.)
}

check for

updates

Citation: Yang, S.; Wang, J.; Li, M.; Yue, H. Research on Intellectualized Location of Coal Gangue Logistics Nodes Based on Particle Swarm Optimization and Quasi-Newton Algorithm. Mathematics 2022, 10, 162. https://doi.org/10.3390/ math10010162

Academic Editors: Quanxin Zhu and Simeon Reich

Received: 23 September 2021 Accepted: 23 December 2021 Published: 5 January 2022

Publisher's Note: MDPI stays neutral with regard to jurisdictional claims in published maps and institutional affiliations.

Copyright: (C) 2022 by the authors. Licensee MDPI, Basel, Switzerland. This article is an open access article distributed under the terms and conditions of the Creative Commons Attribution (CC BY) license (https:// creativecommons.org/licenses/by/ $4.0 /$ )

\begin{abstract}
The optimization of an integrated coal gangue system of mining, dressing, and backfilling in deep underground mining is a multi-objective and complex decision-making process, and the factors such as spatial layout, node location, and transportation equipment need to be considered comprehensively. In order to realize the intellectualized location of the nodes for the logistics and transportation system of underground mining and dressing coal and gangue, this paper establishes the model of the logistics and transportation system of underground mining and dressing coal gangue, and analyzes the key factors of the intellectualized location for the logistics and transportation system of coal and gangue, and the objective function of the node transportation model is deduced. The PSOQNMs algorithm is proposed for the solution of the objective function, which improves the accuracy and stability of the location selection and effectively avoids the shortcomings of the PSO algorithm with its poor local detailed search ability and the quasi-Newton algorithm with its sensitivity to the initial value. Comparison of the particle swarm and PSO-QNMs algorithm outputs for the specific conditions of the New Julong coal mine, as an example, shows that the PSO-QNMs algorithm reduces the complexity of the calculation, increases the calculation efficiency by eight times, saves $42.8 \%$ of the cost value, and improves the efficiency of the node selection of mining-dressing-backfilling systems in a complex underground mining environment. The results confirm that the method has high convergence speed and solution accuracy, and provides a fundamental basis for optimizing the underground coal mine logistics system. Based on the research results, a node siting system for an integrated underground mining, dressing, and backfilling system in coal mines (referred to as MSBPS) was developed.
\end{abstract}

Keywords: integration of mining-dressing-backfilling; coal gangue logistics system; node intelligent location; PSO-QNMs algorithm

\section{Introduction}

\subsection{Study on the Integrated Technologies of Mining-Dressing-Backfilling Systems}

In recent years, with the continuous increase in energy consumption and mining intensity, China's coal mining depth to an average of 10 25 m has sped to the deep extension. Furthermore, deep coal mines need to excavate a large number of rock alleys to meet the needs of the mine production system, surrounding rock stability control, and safe pressure relief mining, which produces a large amount of gangue, which will not only aggravate the contradiction of insufficient lifting capacity of deep shafts, but will also bring infill mining as a sustainable mining technology, which prevents or minimizes the adverse effects of mining coal resources on the environment and other resources from the perspective of mining, with the goal of achieving the best economic, environmental, and social benefits. In recent years, many achievements have been made in backfilling equipment, theories, and 
technologies, in order to further realize that gangue not ascend the shaft and reduce gangue lifting costs, etc. Relevant scholars [1] also put forward the integration technologies of the mining-dressing-backfilling system, which is to establish coal-gangue separation and selection centers, backfilling preparation centers, and gangue pockets in the underground; the coal and gangue products extracted from the underground working face are not lifted to the shaft, but sorted by the underground dressing system, and the sorted gangue is filled in place by the underground backfilling preparation system to realize the gangue backfilling in the extraction area. This technology can achieve safe and efficient recovery of coal resources that cannot be extracted by traditional coal mining methods and improves the resource recovery rate, while improving the effective lifting efficiency of the main shaft and relieving the load of the surface coal washing plant. Moreover, gangue technology can reduce the discharge of gangue on the ground and consequently make full use of gangue to effectively slow ground subsidence, and finally achieve the purpose of protecting the environment and land resources. This is why China strongly advocates these green mining and scientific mining methods.

Experts engaged in this area of research have conducted much research on process optimization and equipment improvement of integrated technologies of the mining-dressingbackfilling system, and on the theory of rock movement patterns caused by this green mining method, mainly from the perspective of traditional mine pressure and formation control and mining technology optimization. Wang Jiachen et al. analyzed the relationship between supports in backfilling mining and surrounding rocks and the movement characteristics of overlying rocks, established the roof load estimation method, used similar simulation and numerical calculation to simulate the process of workface retrieval and gangue backfilling, and verified by backfilling mining examples [2,3]. Zhang Jixiong et al. further proposed the sustainable mining system of "mining-dressing-backfilling $+X$ " in coal mines, revealed the law of mineral pressure manifestation and rock movement control mechanism of solid filling mining, and performed much research on the theory and technology of mining-dressing-backfilling green mining of deep coal resources [4-8]. Tu Shihao developed a theoretical concept of the selective mining technology for the integration technologies of mining-dressing-backfilling systems, and analyzed the critical aspects of "mining-dressing-backfilling + controlling", "mining-dressing-backfilling + extraction", "mining-dressing-backfilling + prevention"," mining-dressing-backfilling + protection", and examined other key mining scientific issues from the perspectives of control backfilling rock movement, stress concentration, fracture field development, and stability of the entry [9]. The results of these studies are relatively mature and have been extensively disseminated in many mines in China.

However, since integrated technologies of the mining-dressing-backfilling system are proposed, it is destined to be a coordinated process of multiple systems in engineering application. On the basis of the existing research to clarify the system composition and structure function of the "mining-dressing-backfilling" system, it is of great significance for the future development of this technology to systematically analyze the operating characteristics of deep underground gangue logistics and the interfeeding linkage relationship. The study of this problem necessarily involves the efficient layout of the "mining-dressing-backfilling" system; first, we must choose the location of the crucial underground "logistics" node, which is the basis to ensure the efficient and coordinated transportation of coal and gangue logistics. The current research results on this issue are relatively few. Wang Jinfeng et al. combined the complex characteristics of a coal mine production logistics system and studied the safety resource allocation, safety evaluation, and production logistics efficiency; further systematic research was conducted on optimization methods to maximize the efficiency of coal mine production logistics systems and rationalize safety resources [10-13]. Based on exploring the key factors affecting the efficiency of the logistics system, Xia Dan et al. used a system dynamics approach to dynamically analyze and predict the efficiency of a complex production logistics system for the integrated technologies of mining-dressingbackfilling systems and calculated the true impact rate of different production steps on 
the production level [14]. Although these studies have dealt with the efficiency of the integrated production and logistics of "mining-dressing-backfilling" systems, they are all from the perspective of macromanagers and have not really achieved substantial research on the logistics node location selection and optimization of the system layout.

\subsection{Study on the Logistics Node Location Selection}

Although there is not much research in the field of coal mining, the location and positioning of key system nodes is very important for the supply chain and logistics transportation system, which need to consider the distance between nodes, cost, and the influence of multiple factors from the perspective of logistics systems, with the continuous development of applied mathematics, increasingly more factors are taken into account, and various siting methods are introduced into logistics node siting in order to select the best location.

The logistics location problem in the supply chain varies in the factors to be focused on in different fields and systems, but multiobjective decision-making oriented to consider multiple factors is an important research topic [15]. Zhang Guofang et al. proposed that the main influencing factors for evaluating the location of logistics nodes are infrastructure platform conditions, basic information platform conditions, and economic and sustainable development conditions of logistics nodes, and in this way subdivided into 28 specific indicators [16]. A multiobjective genetic algorithm (MOGA) was applied in supply chain decision-making for agricultural systems to find the best combination of agricultural inputs that minimize greenhouse gas emissions and maximizes output energy and benefit-cost ratio [17]. The importance of supply and demand on the location of distribution centers is argued [18]. Various factors such as politics, economy, environment, and the enterprise itself are also important for the location of logistics nodes [19]. Considering four criteria in supply chain planning - cost, quality, delivery, and supplier relationship managementa decision method considering quantity discounts and supplier capacity constraints is proposed, and TPSO, PSO, and GA are used for comparative numerical experiments [20]. As research expands, factors such as customer satisfaction, delivery time, service quality, and sustainability are also taken into account [21,22]. Under various operational constraints, cost minimization and profit maximization are the ultimate goals in most supply-chain planning [23,24].

The method of logistics node location selection is evolving as a result of the increasing number of factors to be considered, from the early center-of-gravity method [25-27], it has evolved to multiobjective site selection alternatives including fuzzy integrated analysis [16,28], analytic hierarchy process (AHP) [29-31], and data envelopment analysis (DEA) [32], which can consider more factors and are friendly to some hard-to-quantify factors. While it is computationally difficult to solve larger site planning problems, various heuristic and intelligent algorithms supported by big data and computers are applied to solve the logistics node site selection problem. The alternative location algorithm (ALA) and intelligent algorithms use parallel search techniques to solve the site selection problem, which overcome the difficulty of traditional solution methods and can select the global optimal solution efficiently and accurately. Commonly used algorithms include the ant colony algorithm (ACA) [33,34], genetic algorithm (GA) [35-37], tabu search algorithm (TS) [38], particle swarm optimization algorithm (PSO) [38-40], among others. The common methods and characteristics of logistics node site selection are shown in Table 1.

Recognizing the importance of spatial node layout planning for an integrated coal gangue system of "mining-dressing-backfilling" in the underground, it is necessary to reference logistics node location selection methods that have matured in the field of supply chain and apply them to the integrated technologies of mining-dressing-backfilling systems, and carry out intelligent site selection for the nodes of coal gangue logistics and transportation systems. The integrated production system model of mining, dressing, and backfilling was proposed in the literature [41], and the scientific siting of the nodes of the underground integration technologies of mining-dressing-backfilling systems was studied. 
Due to the complex underground environment of the mine, a three-dimensional logistics space node siting model was developed for the logistics and transportation system under the premise of making appropriate scientific assumptions, and the objective function of multiobjective decision-making was established mainly from the perspective of production efficiency and economy. Furthermore, the PSO algorithm was proposed in solving the objective function. In this paper, the crucial factors of the complicated underground mining and coal gangue transportation system are elaborated from the perspective of logistics rationalization. The coal gangue logistics system location nodes model is further established, and the objective function is defined to identify the vital nodes based on "the highest efficiency and the lowest cost". The traditional evolutionary algorithms, such as the particle swarm algorithm, fully discuss solving this problem, but lack local area search capability, and the results are unstable because of stagnation at the later stage of calculation. The particle swarm and quasi-Newton algorithm (PSO-QNMs) is introduced to design a hybrid algorithm for solving the location of coal gangue logistics nodes by fully taking advantage of the global search capability of the PSO algorithm and the localized and detailed search capability of the quasi-Newton algorithm. The improved algorithm is applied to analyze the issue of coal gangue logistics node siting in a real case: New Julong coal mine.

Table 1. Common methods and characteristics of logistics node location selection.

\section{Key Features}

The distribution of the nodes of the logistics node system is placed on a plane, and the

Center-of-gravity method

Classical solution methods demand and resources of each node are seen as the weight of the point, and the best point for the location of logistics facilities is the center of gravity of the logistics system

Setting the objective function, parameters and variables, making assumptions and constraints simplify, establish a relatively idealized model, and solve it by an appropriate algorithm

A discrete method for evaluating and analyzing alternatives to arrive at the optimal site by

Analytic Hierarchy Process (AHP) establishing an index evaluation system, usually used in conjunction with the fuzzy evaluation method

A system analysis method evolved on the basis of evaluating relative efficiency, adjusting the weight indicators of the evaluation model

Data Envelopment Analysis (DEA)

Multiobjective solving methods dynamically according to the inputs and outputs, evaluating the alternatives from the perspective of the decision unit, independent of the metric and subjective factors of the indicators, and applicable to the site selection decision of multiple input and output problems

It can determine the weight of indicators and quantitative representation of indicators, combine qualitative and quantitative, make a

Fuzzy Integrated Evaluation comprehensive evaluation of a variety of factors, suitable for nondeterministic problem solving, cannot solve the problem of correlation between factors, and the transformation of indicators has a certain degree of subjectivity. 
Table 1. Cont.

Logistics Node Location

Selection Methods

nt Colony Algorithm (ACA)

Genetic Algorithm (GA)

Heuristic and intelligent algorithms

Tabu Search Algorithm (TS)
(PSO)

\section{Key Features}

With fewer setup parameters and good convergence performance, it can generate solutions in a very short time, and is suitable for solving complex logistics node siting problems with great flexibility.

Fast computation and easy combinations with other algorithms.

Easy to understand and implement, strong generality, strong local development ability, fast convergence; based on single solution, and weak group development ability.

Simple operation, fast convergence, does not depend on the strict mathematical properties of the optimization problem itself, can achieve global optimality, and easy to combine with other algorithms.

\section{Coal Gangue Logistics and Transportation Systems in the Integration of Mining-Dressing-Backfilling}

In order to realize the deep underground sorting and in situ filling technology model, an efficient, reliable, intelligent, and economical "coal mining-dressing-backfilling" integrated logistics production system for underground coal mines was established. To study the problem of optimal selection of nodes in the integrated coal gangue logistics production system of "mining-dressing-backfilling" in underground coal mining, the precondition is that the underground mining, sorting, filling, and transportation system links are analyzed separately from the perspective of logistics rationalization. The first and most important is the optimization and rationalization of the logistics system as a whole during the process of completing the underground cycle of mining, sorting, and backfilling from the working face. An underground mining and coal gangue transport system is relatively complex. The core of the two major systems for the coal gangue sorting system consists of the gangue and other waste filling system and the underground mining and coal gangue logistics production, which is to separate coal and gangue in the underground. Gangue is used as the main raw material for underground filling; due to the limited capacity of filling, the flow of gangue produced at the working face and the space relative position of mining and charging will determine the coordinated treatment capacity of mining and filling of the gangue transportation system. Insufficient gangue production will lead to obstruction of the underground filling work, and the surplus of gangue production will cause the excess gangue to be stacked randomly [42]. Therefore, it is necessary to reasonably design the spatial location relationship of key nodes for underground mining, selection, and filling coal gangue logistics systems to ensure efficient and coordinated transportation of mining, selection, and filling. In order to optimize the coal mine production logistics system, the coal production and operation process are transformed into a logistics and transportation process. The integrated coal gangue logistics system of mining, selection, and filling includes two parts: gangue production supply logistics and gangue production logistics. In the transportation part of coal gangue logistics, the normal output of coal gangue is the most fundamental and essential logistical component of the transportation link. In an underground coal mine, the coal and gangue produced from the working face are transported through the complex and extensive transport routes. As there are more logistics nodes in transportation, there will be a certain suspension in the sorting center, the 
filling preparatory center, and the underground gangue silo, based on the efficiency of the whole system, which can be improved by setting a reasonable key node.

\section{Coal Gangue Logistics System Location Nodes Model}

The intelligent selection of nodes in the underground integration of the miningdressing-backfilling system is an optimum solution selected among many solutions to meet the actual engineering background. The establishment and solution of the nonlinear equation system occupies an important position in the optimization problem, especially in the field of industrial engineering, etc. For practical cases, a mathematical model needs to be built and transformed into a system of equations for the problem to be solved. Among many solution methods-particle swarm algorithm, genetic algorithm, ant colony algorithm, Newton's method-search better from the consideration of solution accuracy and convergence, but there are still some defects in the solution process for specific application cases [43-45]. The problem of intelligent output of key nodes in underground integration of mining-dressing-backfilling can actually be regarded mathematically as the problem of large flow, high efficiency, and minimum cost of gangue transportation, by modifying the relevant parameters and changing different constraints, the sum of costs such as construction and transportation is minimized, and the flow rate in the logistics system is maximized. The ultimate goal is to improve the operational efficiency of the coal gangue logistics system. The gangue that is used for filling the working face is partly from the gangue on the surface, and partly from the gangue produced during underground working-face mining and roadway excavation. This is especially important for the large number of rock roadways excavated in deep mining, which gangue is used to enhance the stability of the surrounding rock of the roadway. In the solution of the model, assuming that the surface gangue is transported to the underground gangue silo through the vertical feeding hole, the location of the key nodes of the system is sited from the perspective of maximum logistics and optimal cost, without considering the loss of coal gangue in the transportation process. As shown in Figure 1, the key transportation nodes include six positions, where $I$ is the underground coal-gangue separation and selection center, $J$ is the underground backfilling preparation center, $K$ is the underground gangue pocket, $T$ is the input port, $E$ is the gangue mountain, and $D$ is the shaft coal pocket.

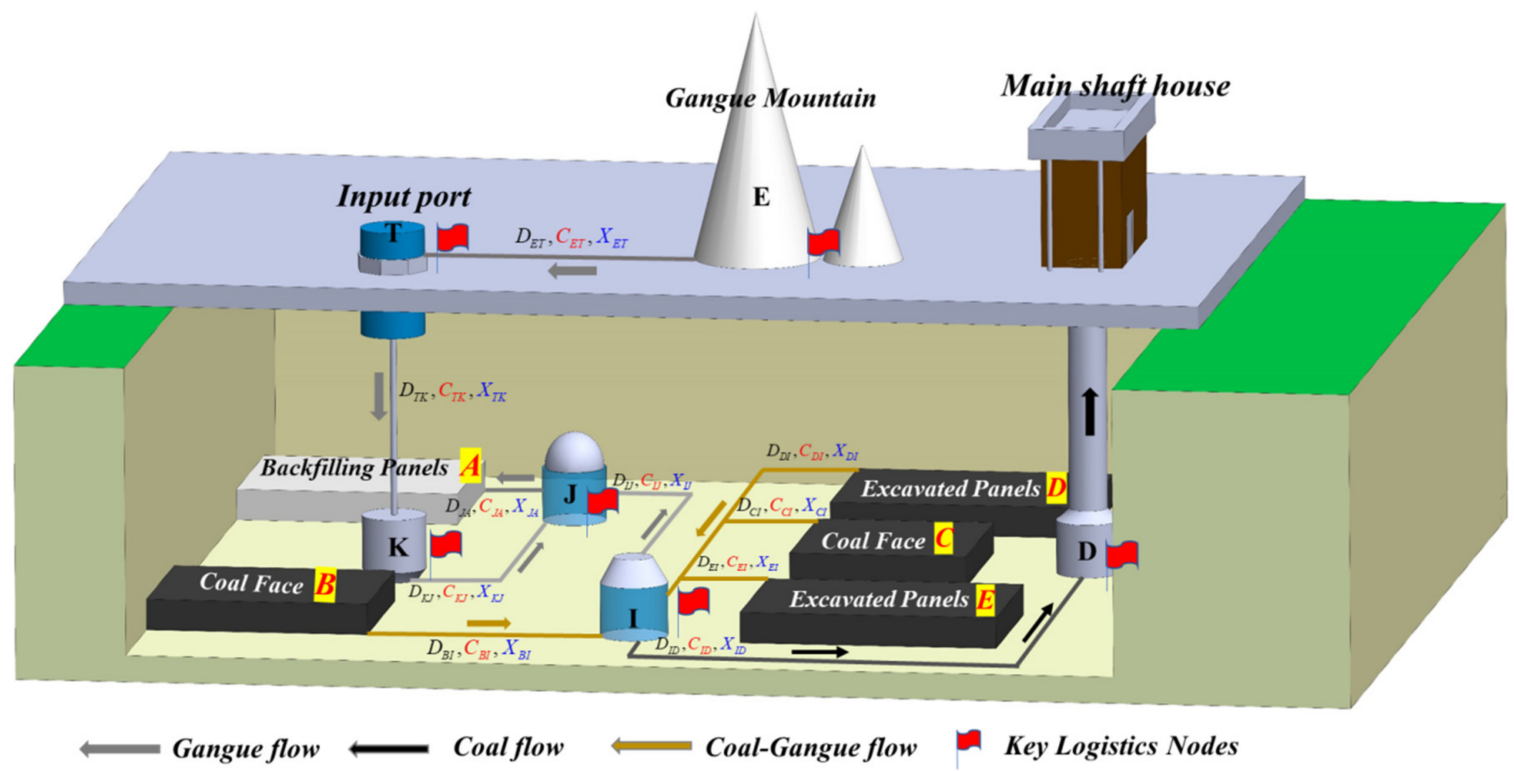

Figure 1. Node transportation model of the coal gangue backfilling system.

From the perspective of economy and logistics, the objective function is established based on the principle of "highest efficiency and lowest cost", i.e., the sum of transportation 
costs between the links is minimized. The objective function is established in Equation (1) and the constraints are given in Equations (2)-(4).

$$
\begin{gathered}
\min Z=\sum_{i \in M} D_{M_{i}, I} C_{M_{i,} I} X_{M_{i}, I}+\sum_{i \in N} D_{N_{i},} C_{N_{i},} X_{N_{i} J}+D_{E, T} C_{E, T} X_{E, T}+ \\
D_{T, K} C_{T, K} X_{T, K}+D_{D, I} C_{D, I} X_{D, I} \\
\sum_{i \in M} X_{M_{i}, I}+\sum_{i \in N} X_{N_{i}, J}=X_{I, J}+X_{I, D} \\
X_{I, J}+X_{K, J}=\sum_{i \in M} X_{J, A} \\
X_{E, T}=X_{T, K}=X_{K, J}
\end{gathered}
$$

where $D_{M_{i}, I}$ indicates position $i$ and $j ; C_{M_{i}, I}$ indicates transportation cost for each unit of coal between position $i$ and $j ; X_{M_{i}, I}$ is the transportation flow rate between position $i$ and $j$; $M_{i}$ is the $i$-th coal mining/excavation working face, where $\left(M_{i}=A, B, C, D, E \cdots\right)$; and $N_{i}$ denote the key logistics nodes in integration of the mining-dressing-backfilling system.

\section{Intelligent Algorithmic Optimization}

\subsection{Particle Swarm Algorithm}

The particle swarm algorithm [22] is a swarm-based random optimization intelligence algorithm that originated from the study of bird feeding behavior, where the simplest and most finite strategy to find food is to search around the bird that is currently closest to the food. The algorithm is an abstraction of solving the objective decision function as the process of searching for the optimal in the decision space in a continuous iteration, which is one of the methods to solve the optimal solution of the multidimensional function. The mathematical description of the algorithm follows. Assuming that the position of particle I in N-dimensional space is represented as a vector: $X_{i}=\left(X_{1}, X_{2}, X_{3}, \ldots \ldots, X_{N}\right)$, and the velocity of the particle motion is represented as a vector: $V_{i}=\left(V_{1}, V_{2}, V_{3}, \ldots \ldots, V_{N}\right)$, then each particle has an adaptation value determined by the objective function and knows the best position experienced by the individual called the individual historical best position, defined as pbest and by its present position; each particle also knows the best position found by all particles in the whole population thus far, which is defined as gbest.

After initializing a group of random particles, the optimal solution is found by iteration. In each iteration, the particles update themselves mainly by tracking pbest and gbest, and the particles will iterate to update their velocity and position according to Equation (5):

$$
\begin{gathered}
V_{i d}(k+1)=\omega V_{i d}(k)+c_{1} r_{1}\left(P_{i d}(k)-X_{i d}(k)\right)+c_{2} r_{2}\left(P_{i d}(k)-X_{i d}(k)\right) ; \\
X_{i d}(k+1)=V_{i d}(k)+V_{i d}(k)
\end{gathered}
$$

where $\omega$ is the inertia weight factor; $c_{1}$ and $c_{2}$ are non-negative constants called the learning factor; and $r_{1}$ and $r_{2}$ are random numbers in $[0,1]$ with independent uniform distribution.

The core code formulas for continuously updating the velocity $\mathrm{New}_{-} v_{i d}$ and position New_ $x_{i d}$ of the particle for each particle motion are Equations (6) and (7), respectively:

$$
\begin{gathered}
\mathrm{New} \_v_{i d}=w * v_{i d}+c_{1} * \operatorname{rand}() *\left(p_{i d}-x_{i d}\right)+c_{2} * \operatorname{rand}\left(p_{g d}-x_{i d}\right) \\
\mathrm{New} \_x_{i d}=x_{i d}+N e w \_v_{i d}
\end{gathered}
$$

where $p_{i d}$ is the individual known optimal solution; $p_{g d}$ is the global known optimal solution; $v_{i d}, x_{i d}$ denote the velocity and position of the particle updated by the last operation in the population, respectively; $w$ is the inertia weight factor; $c_{1}=c_{2}$ is one of the particle learning factors, which usually is valued between 0 and 2; and rand() is a random number within $(0,1)$. To reduce the possibility of particles leaving the search space during the search process, $V$ is usually limited to a certain range. 
The PSO is an efficient parallel search algorithm that retains a population-based global search strategy with a relatively simple operational model that preserves the individual historical extremes of each particle, which has been applied to the output of the key node location in the coal gangue logistics and transportation system of integrated underground mining, dressing, and backfilling The author references particle swarm algorithm in the literature to solve the node siting for the integrated logistics of the mining-dressingbackfilling system, and some of the core codes have also been reflected in the literature, the algorithm initially realized the automatic output of coal gangue logistics node siting under the role of complex factors. However, it was found in subsequent application that the algorithm lacks the ability of fine search in a local area, and the phenomenon of convergence stagnation that often occurs in the later stage of the search is not very sensitive to the population size and cannot obtain an unique and accurate solution.

\subsection{Quasi-Newton Methods Algorithm}

The quasi-Newton methods (QNMs) [46-50] were first described by the American physicist Davidson in the mid-1950s, and shortly thereafter it was proved by the operational scientists Fletcher and Powell to be both faster and more stable than the algorithms available at that time. In recent years, the QNMs have become an important research area for algorithms to solve both constrained and unconstrained optimization problems. The QNMs do not need to calculate the Hesse array of the objective function in the computational process as does the Newton method, yet it can have the same efficacy in some sense as when using the Hesse array, and has a second-order convergence speed. This not only simplifies the computational process, but also ensures algorithm convergence speed. Therefore, in recent decades, the QNMs are one of the most important methods for solving nonlinear systems of equations and optimization problems. The QNMs program code is shown in Equation (8):

$$
\text { function }[k, x, v a l]=b f g s\left(f u n, g f u n, x_{0} \text {, varargin }\right)
$$

where $k$ is the number of iterations; $x, v a l$ is the approximate optimal site and the optimal value, respectively; $f u n, g f u n$ is the objective function and its gradient, respectively; $x_{0}$ is the initial site; and varargin is the input variable parameter.

The main characteristics of the QNMs are simple internal update rules, high accuracy, strong numerical stability, and fast convergence. However, the selection of the initial value of the method is challenging; if a random value is used for the solution, then in actual engineering background application it extremely difficult attain convergence. Therefore, the convergence depends on the selection of the initial value; thus, it is very important to provide an optimal initial value for QNMs.

\subsection{Particle Swarm and Quasi-Newton Algorithm}

In view of the poor local search ability of the PSO algorithm and the sensitivity of the QNMs algorithm to the initial value, in order to improve the localization accuracy and convergence, combining the characteristics of the two algorithms, a particle swarm optimization algorithm based on quasi-Newton algorithm (PSO-QNMs algorithm), is designed to precisely optimize the nodes of the coal and gangue system to achieve the effect of making full use of the advantages of the two algorithms. The process of the PSO-QNMs algorithm is shown in Figure 2. Firstly, the PSO algorithm is used to search the problem in a wide range within the feasible solution area to find the optimal algorithm to a certain extent, and to provide a good initial point for the QNMs algorithm, which is used as the initial value of the QNMs algorithm for continuous iteration, then the QNMs algorithm was used to search precisely until a more precise root of the equation is found. The program code is shown in Equation (9):

$$
\begin{aligned}
\text { function } y= & f \text { funadd }(n, x, \text { fixed point, } \cos t, \text { trafficflow }) \\
& \text { function } y=\text { funadd } G r a(x)
\end{aligned}
$$




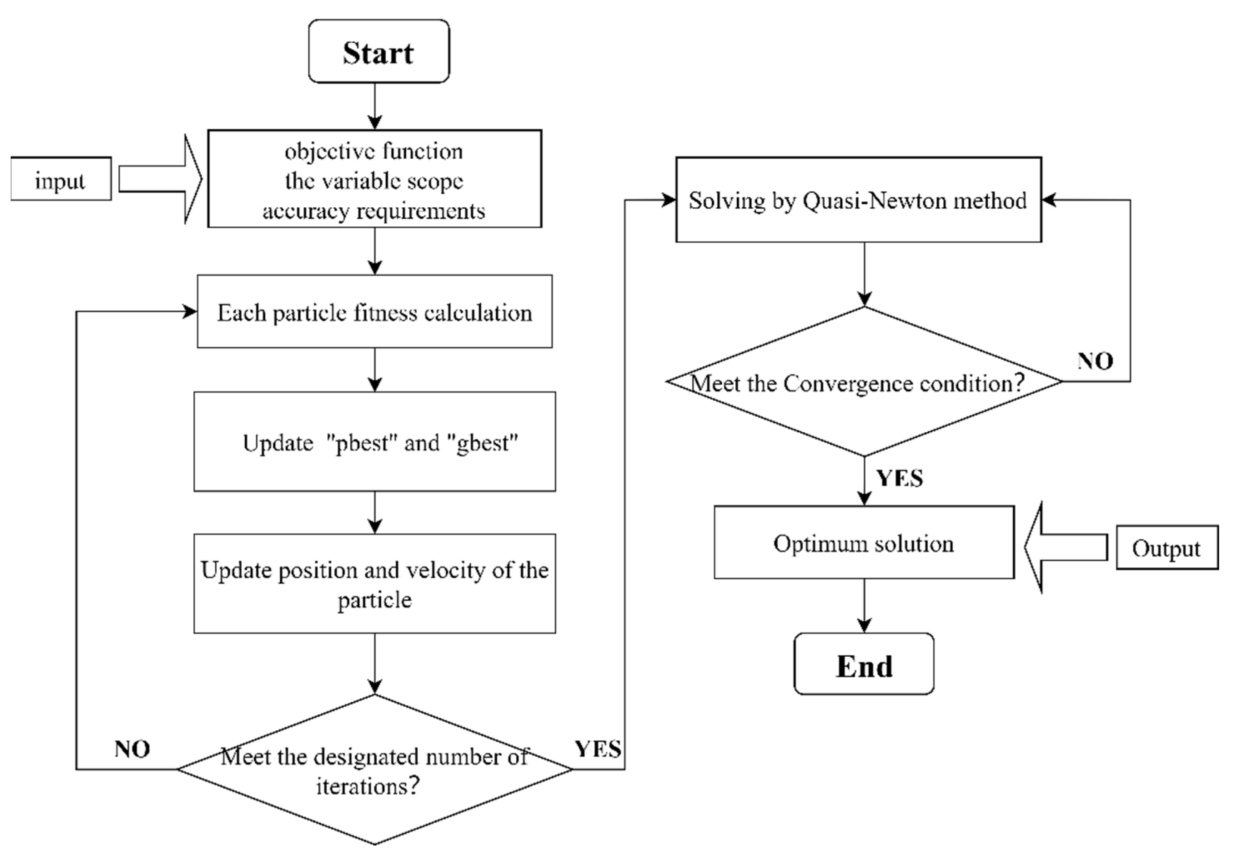

Figure 2. Flow chart of the PSO-QNMs hybrid algorithm.

The PSO-QNMs [25] runs the particle swarm optimization algorithm $\mathrm{M}$ generation at the initial stage (population size is $\mathrm{m}$ ), reaches the termination condition, outputs the current global optimal individual (defined in the PSO algorithm as "zbest") and gives this value to the initial value of Newton method, as indicated in Equation (10):

$$
x_{0}=\text { zbest }
$$

where $x_{0} \in \mathrm{R}^{\mathrm{n}}$, termination of the error $0 \leq \varepsilon \leq 1$. The initial positive definite matrix $H_{0} \in R^{n \times n}, k:=0$; if $\left\|g_{k}\right\| \leq \varepsilon$, then the operation is stopped and the output $x_{k}$ is taken as an approximate minimum point; the direction of calculation and search $d_{k}=-H_{k} g_{k}$, and $\alpha_{k}$ is solved by linear search along direction $d_{k} ; x_{k+1}:$ is expressed as $x_{k+1}:=x_{k}+\alpha_{k} d_{k}$. Calibration $H_{k}$ produces $H_{k+1}$, and $k=k+1$ iterations are performed. Part of the core program code of the PSO-QNMs algorithm is shown in Equation (11):

fminunc_options = optimoptions(@fminunc,' Algorithm', quasi - newton' , MaxFunEvals', 100000,

$$
\text { 'PlotFcns', @optimplotfval); }
$$

[Gra_best,fval_Gra, exitflag_Gra, output_Gra] = fminunc(@funadd_Gra,x0,fminunc_options)

\section{Case Analysis}

\subsection{Background}

This algorithm comparison uses the Xinjulong coal mine of Shandong Energy Group as the basic background for engineering application to simulate the intellectualized location of coal gangue logistics nodes of integrated system for "mining-dressing-backfilling" in the underground. The working face of the Xinjulong coal mine, which is mainly a fully mechanized coal caving and backfilling face, and the production of the double mining district is mainly at the same gallery level. The coal seam thickness of the $1302 \mathrm{~N}-2 \#$ backfilling face is $2.2-3.63 \mathrm{~m}$, the average coal thickness is $2.73 \mathrm{~m}$, the mining coefficient is 1 , and the coal seam variation coefficient is $11.9 \%$, which belongs to a medium thick coal seam with simple structure and stable thickness within the mining range of the working face. The $1302 \mathrm{~N}-2$ \# backfilling face is located north of the $1 \#$ direct track rise at the -810 level, east is the $1302 \mathrm{~N}-1$ \# gob, west is the unprepared $1303 \mathrm{~N}-1$ \# backfilling face, south is the village protective coal pillar, and north is the 1302N\#gob protective coal pillar. 
The gangue for backfilling in the working face comes from the fully mechanized caving face, the fully mechanized backfilling panel, and the excavated panel produced at the same time. In the simulation, three coal mining faces and two tunneling faces are set. The gangue produced from the $1302 \mathrm{~N}-2$ \# backfilling face goes through the sieving and smashing system; the excavated gangue is directly transported to the gangue pocket. The raw coal mixed with coal and gangue is transferred to the coal-gangue separation system, and the cleaned coal after separation is moved to the transportation roadway through the loading station of the transportation roadway, and finally is lifted to the ground through the main shaft. The gangue is transported to the gangue pocket of the first mining wing for storage along the gangue transport roadway, and transported to the backfilling face for gob through the 1302N-2\#tailentry and the return-air rise. The coal gangue logistics and transportation system in the integrated mining-dressing-backfilling system at the Xinjulong coal mine is shown in Figure 3.

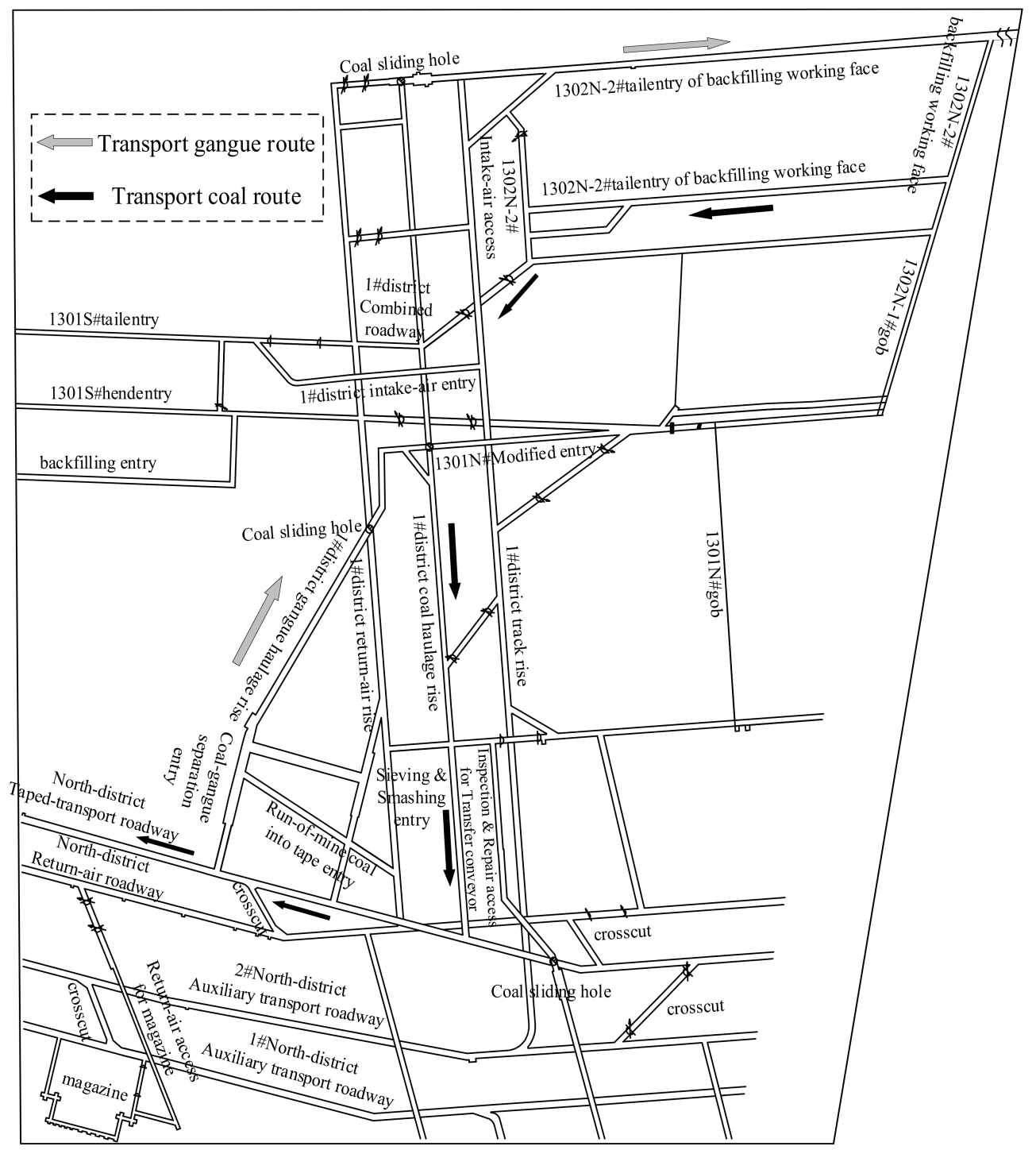

Figure 3. Schematic diagram of coal gangue logistics and transportation system in the Xinjulong coal mine.

The PSO algorithm and the PSO-QNMs algorithm are used to carry out the case study. In this calculation, the gangue selection rate is 95\%; the fixed coordinates of transportation links for each logistics system and the flow parameters of working face are given in Table 2. 
Furthermore, based on the project background, the input of ground gangue is also taken into account in this analysis.

Table 2. Input parameters.

\begin{tabular}{|c|c|c|c|c|c|c|c|}
\hline Parameter & Coal Face 1\# & Coal Face 2\# & Coal Face 3\# & $\begin{array}{c}\text { Excavated } \\
\text { Panel } \\
\text { 1\# }\end{array}$ & $\begin{array}{c}\text { Excavated } \\
\text { Panel } \\
\text { 2\# }\end{array}$ & $\begin{array}{c}\text { Shaft Coal } \\
\text { Pocket }\end{array}$ & $\begin{array}{l}\text { Gangue } \\
\text { Mountain }\end{array}$ \\
\hline $\begin{array}{c}\text { fixed } \\
\text { coordinates }\end{array}$ & $(1000,3000,-800)$ & $(3000,1000,-900)$ & $(2000,2000,-850)$ & $(1000,1500,-800)$ & $(1500,1000,-950)$ & $(1500,2000,-750)$ & $(1000,2000,-0)$ \\
\hline flow $/(t / h)$ & 230 & 220 & 240 & 200 & 210 & - & - \\
\hline $\begin{array}{c}\text { gangue } \\
\text { percentage }\end{array}$ & 0.32 & 0.3 & 0.3 & 0.35 & 0.33 & - & - \\
\hline
\end{tabular}

\subsection{Results}

In this paper, three groups of experiments are designed. The PSO algorithm and the PSO-QNMs algorithm are set in each group, and the values of the output results are analyzed, based on the two solution algorithms. In the solution process, the same initial value is given to the two intelligent optimization algorithms. In the process of the PSO algorithm, after several iterative solutions, the fitness value of the node particles after each operation was compared with the population optimal solution in the particle swarm. The resulting optimal value was used to replace the population optimal value, and the particle with the most adaptive value was obtained; that is, the objective result of the optimal function was obtained and the calculation was then terminated. In the process of the PSO-QNMs algorithm, the preliminary calculation steps are the same as the PSO algorithm, except that the termination condition of the algorithm is when the calculated gradient value $g_{k}$ meets the termination error $\varepsilon$ of the algorithm. The two algorithms were programmed and solved by using MATLAB. The data of the three groups of experimental results are shown in Table 3. After the program operation, the iterative graphs of the two intelligent optimization algorithms are output, as shown in Figure 4. The spatial location relationship diagrams of logistics nodes of the three groups of intelligent optimization algorithms are output, as shown in Figures 5-7.

Table 3. Simulation experiment results.

\begin{tabular}{|c|c|c|c|c|c|c|c|c|}
\hline Experiments & Algorithm & & $\begin{array}{c}\text { Separation } \\
\text { and } \\
\text { Selection } \\
\text { Centers }\end{array}$ & $\begin{array}{c}\text { Backfilling } \\
\text { and Prepa- } \\
\text { ration } \\
\text { Centers }\end{array}$ & $\begin{array}{l}\text { Gangue } \\
\text { Pockets }\end{array}$ & $\begin{array}{c}\text { Optimal } \\
\text { Values } \\
I \times 10^{8}\end{array}$ & $\begin{array}{l}\text { Maximum } \\
\text { Number of } \\
\text { Iterations }\end{array}$ & $\begin{array}{c}\text { Operation } \\
\text { Time } \\
\text { /s }\end{array}$ \\
\hline \multirow{7}{*}{ Group 1} & \multirow{3}{*}{ PSO } & \multirow{3}{*}{$X Y Z$} & 1675.449 & 865.714 & -114.715 & \multirow{3}{*}{3.293} & \multirow{3}{*}{2670} & \multirow{3}{*}{8.840} \\
\hline & & & 1676.430 & 1676.819 & 639.201 & & & \\
\hline & & & -676.581 & -331.045 & -232.421 & & & \\
\hline & \multirow{4}{*}{$\begin{array}{l}\text { PSO- } \\
\text { QNMs }\end{array}$} & \multirow{4}{*}{$X Y Z$} & 1499.999 & 1460.048 & 1460.047 & \multirow{4}{*}{1.846} & \multirow{4}{*}{53} & \multirow{4}{*}{1.148} \\
\hline & & & 2000.000 & 2037.973 & 2037.973 & & & \\
\hline & & & -750.000 & -717.835 & -717.835 & & & \\
\hline & & & 1133.104 & 678.695 & 888.8732 & & & \\
\hline \multirow{5}{*}{ Group 2} & \multirow[t]{2}{*}{ PSO } & \multirow[t]{2}{*}{$X Y Z$} & 1765.071 & 697.2961 & 661.397 & \multirow[t]{2}{*}{3.359} & \multirow[t]{2}{*}{2420} & \multirow[t]{2}{*}{9.633} \\
\hline & & & -816.158 & -866.207 & -768.231 & & & \\
\hline & \multirow{4}{*}{$\begin{array}{l}\text { PSO- } \\
\text { QNMs }\end{array}$} & \multirow{3}{*}{$X Y Z$} & 1500.000 & 1454.982 & 1454.979 & \multirow{4}{*}{1.846} & \multirow{4}{*}{52} & \multirow{4}{*}{1.098} \\
\hline & & & 2000.000 & 2040.568 & 2040.568 & & & \\
\hline & & & -750.000 & -715.002 & -714.999 & & & \\
\hline \multirow{6}{*}{ Group 3} & & \multirow{3}{*}{$X Y Z$} & 1592.962 & 298.096 & 270.873 & & & \\
\hline & \multirow[t]{2}{*}{ PSO } & & 1894.860 & 1090.314 & 1231.951 & \multirow[t]{2}{*}{3.019} & \multirow[t]{2}{*}{2870} & \multirow[t]{2}{*}{8.681} \\
\hline & & & -753.395 & -225.351 & -376.964 & & & \\
\hline & \multirow{3}{*}{$\begin{array}{l}\text { PSO- } \\
\text { QNMs }\end{array}$} & \multirow{3}{*}{$X Y Z$} & 1500.000 & 1456.274 & 1456.273 & \multirow{3}{*}{1.846} & \multirow{3}{*}{60} & \multirow{3}{*}{1.269} \\
\hline & & & 2000.000 & 2039.312 & 2039.312 & & & \\
\hline & & & -749.999 & -715.927 & -715.927 & & & \\
\hline
\end{tabular}




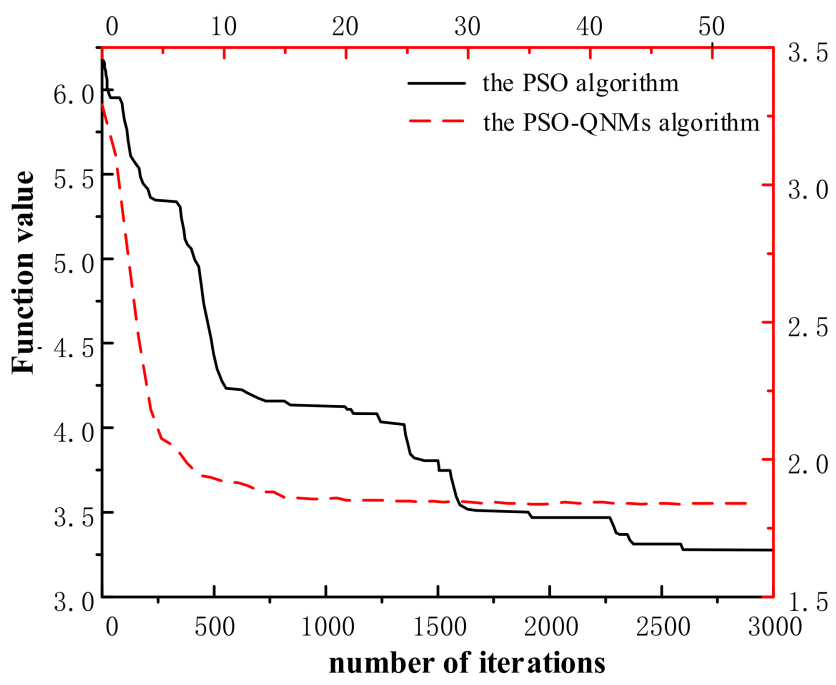

(a)

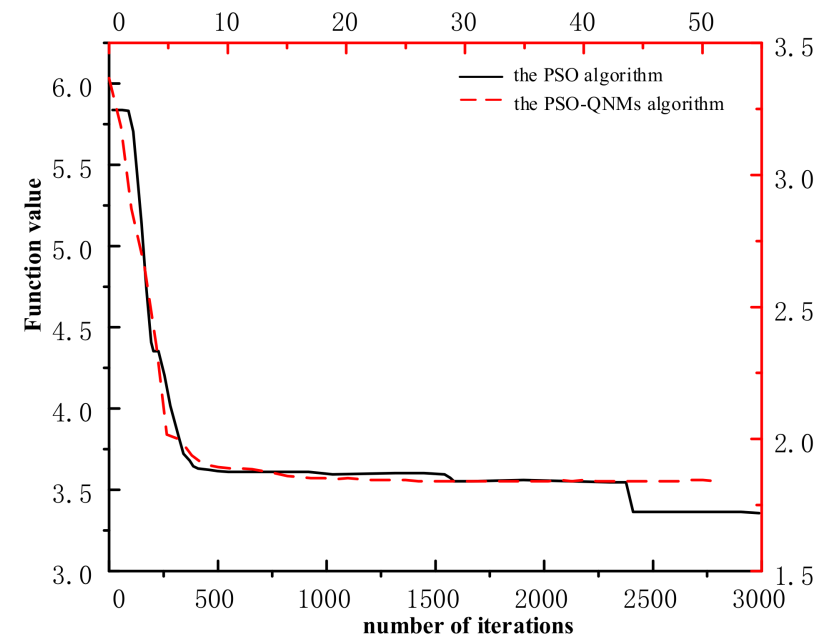

(b)

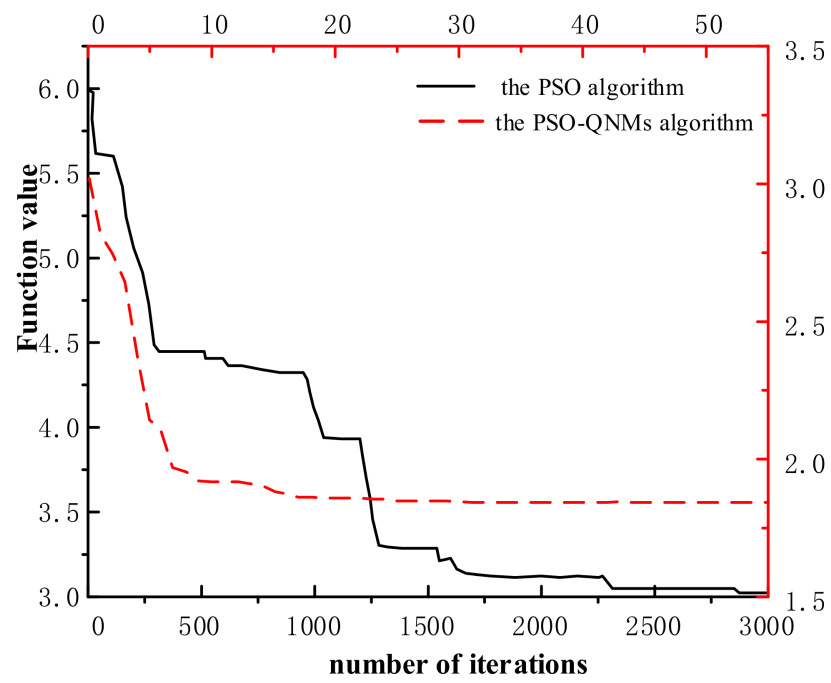

(c)

Figure 4. Intelligent optimization method iteration curve: (a) group 1, (b) group 2, (c) group 3. 


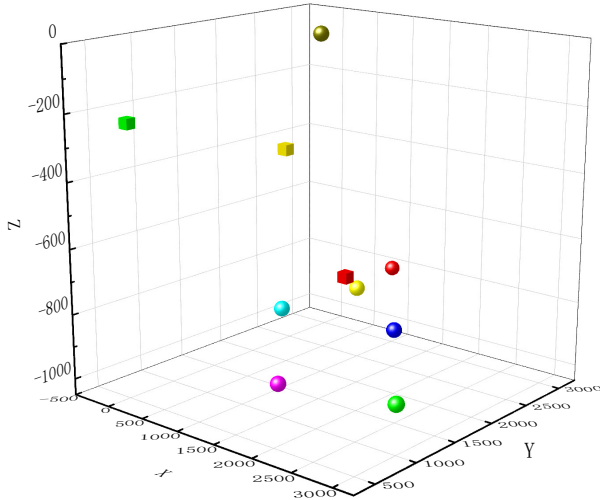

(a)

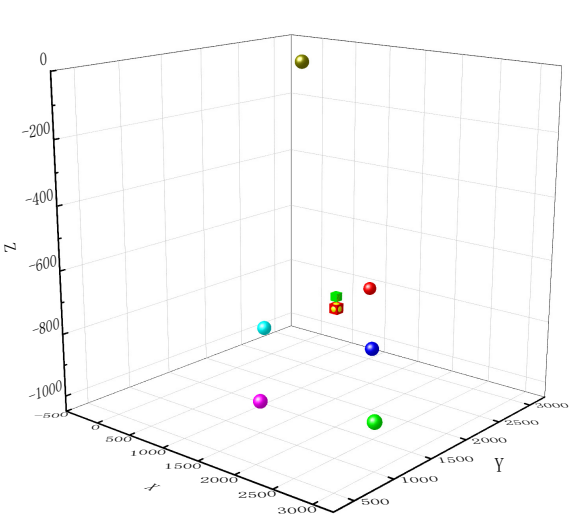

(b)

\section{Coal face 1\# \\ Coal face 2\# \\ Coal face 3\# \\ Q Excavated Panel 1\# \\ CExcavated Panel 2\# \\ Shaft coal pocket \\ Gangue mountain \\ Separation\& selection \\ Backfilling preparation \\ center \\ Gangue pocket}

Figure 5. The spatial position relationship diagram of the logistics nodes (group 1): (a) PSO algorithm, (b) PSO-QNMs algorithm.

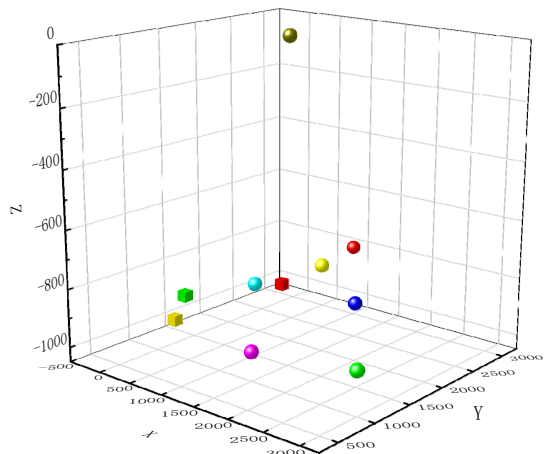

(a)

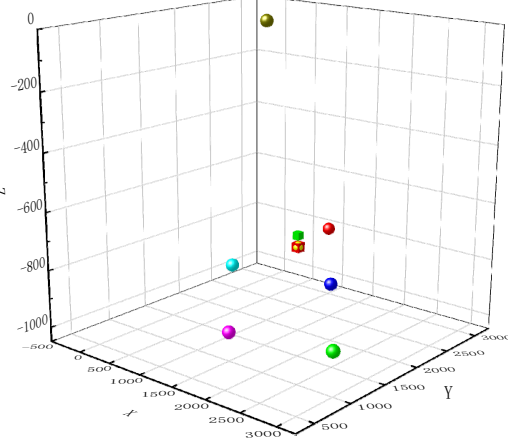

(b)

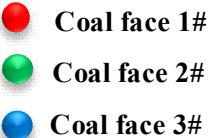

Coal face $3 \#$

Qxcavated Panel 1\#

Excavated Panel 2\#

Shaft coal pocket

Gangue mountain

Separation and selection center

Backfilling preparation center

Gangue pocket

Figure 6. The spatial position relationship diagram of the logistics nodes (group 2): (a) PSO algorithm, (b) PSO-QNMs algorithm.

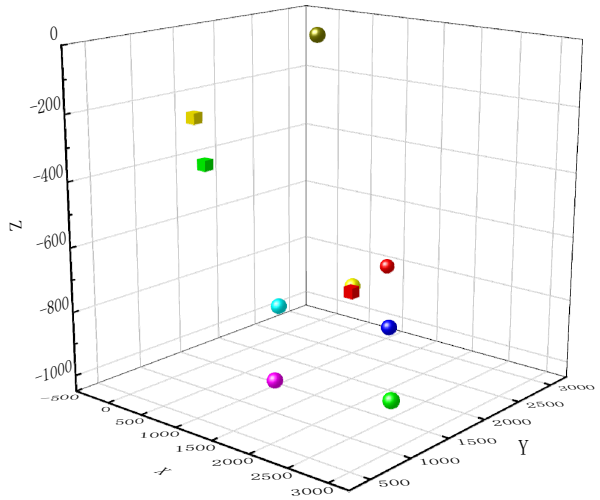

(a)

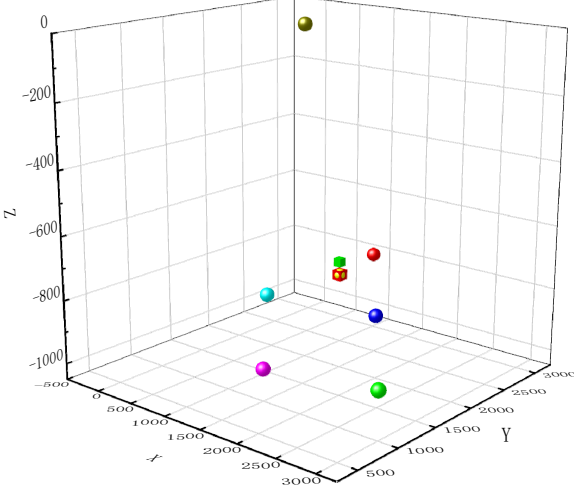

(b)

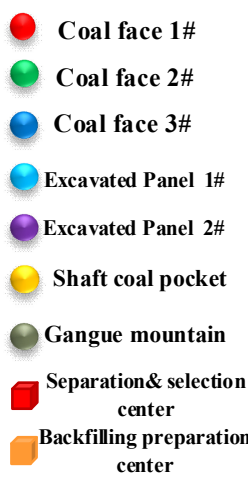

Gangue pocket

Figure 7. The spatial position relationship diagram of the logistics nodes (group 3): (a) PSO algorithm, (b) PSO-QNMs algorithm. 


\subsection{Analysis}

(1) Combined with the simulation results (Table 2) and the intelligent optimization algorithm iterative graph (Figure 4), it can be concluded that the PSO-QNMs algorithm, compared with the PSO algorithm, has stronger ability to converge to the global optimal solution, and its convergence speed is significantly higher than that of PSO. The solution time of the whole system operation is about one-eighth of that of the latter. Therefore, the PSO-QNMs algorithm reduces the computational complexity and ensures the global convergence of the algorithm when solving the coal gangue logistics nodes in the underground integration technologies of the mining-dressingbackfilling system. At the same time, when the number of iterations of PSO algorithm is larger, the accuracy of the optimal solution is relatively higher, and then the initial value assigned to the QNMs is better, and the PSO-QNMs algorithm can give full play to its global fine search performance, and the search effect of the optimal solution is better.

(2) According to the optimal function value output from the simulation experiment results in Table 2, it can be concluded that the PSO-QNMs reduces the cost value by about $42.8 \%$ compared with the PSO algorithm, indicating that the former has a good approximation effect on the extreme value of the objective function of the coal gangue logistics nodes model, and its accuracy can be improved by $100 \%$.

(3) Comparing the three groups of the intelligent optimization algorithm logistics node location diagram (Figures 5-7), it can be concluded that the PSO selects nonunique coal gangue logistics system location nodes, and the output is not stable. However, the PSO-QNMs algorithm is able to select basically similar but extremely stable results. Therefore, the PSO-QNMs algorithm is more accurate and stable than the PSO, which also demonstrates the superiority of the PSO in solving the nodes in the coal and gangue transportation system of mining and separation within a complex environment.

\section{Node Location Decision System for Integration Technologies of the Mining-Dressing-Backfilling System}

Based on the research results, a node siting system for an integrated underground mining-dressing-backfilling system in coal mines, referred to as MSBPS, was developed as shown in Figure 8. This is a logistics system node siting output system that is closely related to the research content of integrated deep underground mining, dressing, and backfilling. It only needs to input the spatial location coordinates of specific known nodes and the unit cost between each link, and calculates the solution independently to output the requested optimal node location information, i.e., it can output the spatial coordinates of node locations with one key. At the same time, it can obtain the three-dimensional spatial location relationship between the requested node location and specific known nodes, with a data export function and Chinese and English interface operation, which greatly simplifies the complexity of outputting node locations. It provides a fast, convenient, and accurate image and data export platform for locating nodes deep underground in an integrated mining, dressing, and backfilling logistics system, and is an effective three-dimensional dynamic system platform design tool for conducting further research on deep mining, dressing, and backfilling. 

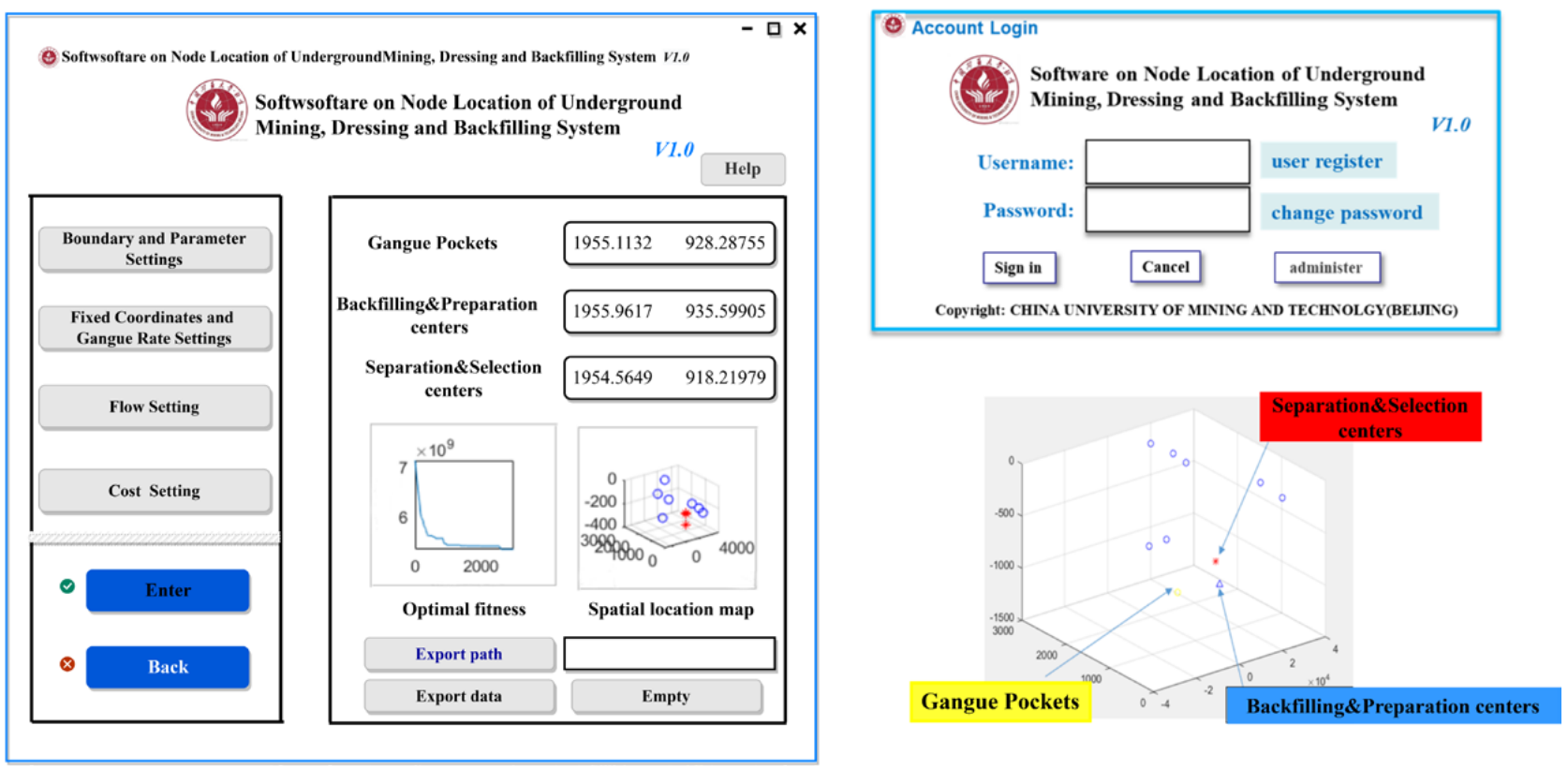

Figure 8. Main Interfaces of the node location decision system.

\section{Conclusions}

Based on the site selection of coal and gangue logistics nodes in the integration of mining, separation, and filling, this paper provides an in-depth study and proposes an improvement in the precise intelligent optimization algorithm of nodes in underground integration technologies of a mining-dressing-backfilling system. Based on the poor local search ability of the PSO algorithm and the instability of node output results, the PSOQNMs algorithm was proposed. This algorithm realized a fast and fine search of nodes in the integrated logistics system of coal mining-dressing-backfilling under the action of complex factors. The following main conclusions were obtained:

(1) Based on the complex coal mine environment in China, the relative positions of coalgangue separation and selection center, backfilling preparation center, and gangue pocket are provided by combining the following components: practical engineering background; mining-dressing-backfilling underground transportation system analysis; construction of coal gangue system node location model; and "high efficiency, lowest cost" as the principal function of a variety of intelligent optimization algorithms. It has practical guiding value in future use of the optimization algorithm of node intelligence for complex underground systems.

(2) Based on the poor global convergence of the PSO algorithm, the PSO-QNMs algorithm is proposed. The results of several groups of simulation experiments have shown that the PSO-QNMs algorithm has stronger convergence than the PSO algorithm in solving the node position of the mining-dressing-backfilling system in complex environment, and the whole operation time is only $1 / 8$ of that of the PSO.

(3) Based on the poor global convergence of the PSO algorithm, the PSO-QNMs algorithm is proposed. The results of several groups of simulation experiments have shown that the PSO-QNMs algorithm has stronger convergence than the PSO algorithm in solving the node position of the mining-dressing-backfilling system in a complex environment, and the whole operation time is only one-eighth that of the PSO algorithm.

(4) In terms of objective function value, the PSO-QNMs algorithm reduces the cost value by about $42.8 \%$ compared with the PSO, which optimizes the objective function value, and improves the node optimization efficiency of the mining-dressing-backfilling system within a complex underground environment. 
(5) By comparing the performance of the PSO and PSO-QNMs algorithms in the spatial position coordinates and optimal solution of objective function of logistics nodes, it is further confirmed that the PSO-QNMs algorithm is of high precision and provides stable experimental output results. The superiority of the PSO-QNMs algorithm to solve the intellectualized location of coal gangue logistics nodes under complex environment is proven.

(6) Based on the research results, a node siting system for integrated underground mining, processing, and charging systems in coal mines (referred to as MSBPS) was developed, which is an effective tool for further research on three-dimensional dynamic platform design for an integrated deep mining, processing, and charging system.

Author Contributions: S.Y.: conceptualization, data curation, funding acquisition, methodology, project administration, investigation, software, writing-original draft, and writing-review and editing; J.W.: data curation, formal analysis, investigation, software, and writing—original draft; M.L.: data curation, formal analysis, investigation, and software; H.Y.: formal analysis and investigation. All authors have read and agreed to the published version of the manuscript.

Funding: This research was funded by the National Key Research and Development Program of China (no. 2018YFC0604701).

Institutional Review Board Statement: Not applicable.

Informed Consent Statement: Not applicable.

Data Availability Statement: All data used during the study appear in the submitted article.

Conflicts of Interest: The authors declare no conflict of interest.

\section{References}

1. He, Q. Research on Key Technology of Integration of Mining, Dressing, Backfilling and Mining in Underground Coal Mine. Ph.D. Thesis, China University of Mining and Technology, Xu Zhou, China, 2014.

2. Wang, J.; Yang, S. Research on support-rock system in solid backfill mining methods. J. China Coal Soc. 2010, 35, 1821-1826.

3. Wang, J.; Yang, S.; Yang, B. Simulation experiment of overlying strata movement features of longwall with gangue backfill mining. J. China Coal Soc. 2012, 37, 1256-1262.

4. Zhang, J. Sthdy on Strata Movement Controlling by Raw Waste Backfilling with Fully-Mechanized Coal Winning Technology and Its Applications. Ph.D. Thesis, China University of Mining \& Technology, Xu Zhou, China, 2008.

5. Zhang, J.; Zhou, Y.; Huang, Y. Integrated technology of fully mechanized solid backfill mining. Coal Sci. Technol. 2012, 40, 10-13.

6. Zhang, J.; Zhang, Q.; Ju, F.; Zhou, N.; Li, M.; Sun, Q. Theory and technique of greening mining integrating mining, separating and backfilling in deep coal resources. J. China Coal Soc. 2018, 43, 377-389.

7. Zhang, J.; Zhang, Q.; Ju, F.; Zhou, N.; Li, M.; Zhang, W. Practice and technique of green mining with integration of mining, dressing, backfilling and $X$ in coal resources. J. China Coal Soc. 2019, 44, 64-73.

8. Miu, X.; Zhang, J. Key technology of integration of coal mining gangue washing backfilling and coal mining. J. China Coal Soc. 2014, 39, 1424-1433.

9. Tu, S.; Hao, D.; Li, W.; Liu, X.; Miao, K.; Yang, Z.; Ban, J. Construction of the theory and technology system of selective mining in "mining, dressing, backfilling and X" integrated mine. J. Min. Saf. Eng. 2020, 37, 81-92.

10. Wang, J.; Zhu, Y.; Feng, L. Research on Resource Allocation Efficiency of Coal Mine Production Logistics System. Coal Eng. 2014, $46,114-117$.

11. Wang, J.; Du, X.; Feng, L.; Zhai, X. Safety resource allocation model of coal mine production logistics system using stochastic programming. China Saf. Sci. J. 2015, 25, 16-22.

12. Wang, J.; Zhai, X.; Feng, L. Efficiency Optimization of Coal Mine Production Logistics Under Safety Hard Constraint. Chin. J. Manag. Sci. 2014, 22, 59-66.

13. Jia, Y.; Du, X.; Wang, J.; Feng, L. Research of security resource allocation model of coal mine production logistics system based on maximum information entropy. Ind. Mine Autom. 2016, 42, 33-37.

14. Xia, D.; Wang, X. Efficiency simulation of integrated production logistics system for underground coal mining, dressing and filling. China Min. Mag. 2020, 29, 75-81.

15. Dou, Z.; Shao, Y.; Yuan, Z.; Ji, M. Review on Logistics Node Location Selection. Logist. Eng. Manag. 2020, $42,1-4$.

16. Zhang, G.; Bao, F. Research on Logistics Centre with Fuzzy Synthesis Evaluation of Entropy Weights Model. J. Wuhan Univ. Technol. 2005, 7, 91-93. 
17. Shamshirband, S.; Khoshnevisan, B.; Yousefi, M.; Bolandnazar, E.; Anuar, N.B.; Wahab, A.W.A.; Khan, S.U.R. A multi-objective evolutionary algorithm for energy management of agricultural systems-A case study in Iran. Renew. Sustain. Energy Rev. 2015, 44, 457-465. [CrossRef]

18. Chu, T.C.; Lai, M.T. Selecting distribution centre location using an improved fuzzy MCDM approach. Int. J. Adv. Manuf. Technol. 2005, 26, 293-299. [CrossRef]

19. Zak, J.; Weglinski, S. The selection of the logistics center location based on MCDM/A methodology. In Proceedings of the 17th Meeting of the EURO-Working-Group on Transportation (EWGT), Seville, Spain, 2-4 July 2014; pp. 555-564.

20. Che, Z.H.; Chiang, T.-A.; Che, Z.-G. Using analytic network process and turbo particle swarm optimization algorithm for non-balanced supply chain planning considering supplier relationship management. Trans. Inst. Meas. Control. 2012, 34, 720-735. [CrossRef]

21. Zhang, R.; Wang, Z. Bi-level Programming Model and Solution Algorithm for Layout of Terminals of City Distribution. J. Tongji Univ. (Nat. Sci.) 2012, 40, 1035-1040.

22. Guo, X.; Wang, Y. Multi-objective Model for Logistics Distribution Programming Considering Logistics Service Level. J. Southwest Jiaotong Univ. 2012, 47, 874-880.

23. Cui, L.X. Joint optimization of production planning and supplier selection incorporating customer flexibility: An improved genetic approach. J. Intell. Manuf. 2016, 27, 1017-1035. [CrossRef]

24. Griffis, S.E.; Bell, J.E.; Closs, D.J. Metaheuristics in Logistics and Supply Chain Management. J. Bus. Logist. 2012, 33, 90-106. [CrossRef]

25. Niu, D.; Wu, J. A Case Study of Multiple Gravity Method Site Selection. Mark. Manag. Rev. 2021, 152-153. [CrossRef]

26. Velazquez-Marti, B.; Fernandez-Gonzalez, E. Mathematical algorithms to locate factories to transform biomass in bioenergy focused on logistic network construction. Renew. Energy 2010, 35, 2136-2142. [CrossRef]

27. Almetova, Z.; Shepelev, V.; Shepelev, S. Cargo Transit Terminal Locations According to the Existing Transport Network Configuration. In Proceedings of the 2nd International Conference on Industrial Engineering (ICIE), Chelyabinsk, Russia, 19-20 May 2016; pp. 1396-1402.

28. Huang, M.; Cui, Y.; Yang, S.; Wang, X. Fourth party logistics routing problem with fuzzy duration time. Int. J. Prod. Econ. 2013, 145, 107-116. [CrossRef]

29. Fang, L.; He, J. Combining the Analytic Hierarchy Process and Goal Programming for Location M odel of Emergency Systems. Syst. Eng.-Theory Pract. 2003, 62, 116-120.

30. Bi, K.; Yang, M.; Zahid, L.; Zhou, X. A New Solution for City Distribution to Achieve Environmental Benefits within the Trend of Green Logistics: A Case Study in China. Sustainability 2020, 12, 8312. [CrossRef]

31. Xi, S.-E. Evaluation of Nodes Importance in of Highway Transportation Hubs Planning Based on Fuzzy-AHP. In Proceedings of the 2nd International Conference on Civil Engineering and Transportation (ICCET 2012), Guilin, China, 27-28 October 2013; pp. 1181-1187.

32. Zhang, S.; Cui, R. Logistics Efficiency Network Spatial Structure Based on Coastal City Shandong. J. Coast. Res. 2020, 322-327. [CrossRef]

33. Piao, C.; Hu, H.; Zhang, Y. Logistics distribution vehicle path planning research. In Proceedings of the IEEE International Conference on Artificial Intelligence and Information Systems (ICAIIS), Dalian, China, 20-22 March 2020; pp. 396-399.

34. Zou, Q. Research on Hybrid Hub-Spoke Express Network Based on Multiple Transportation Modes. In Proceedings of the IEEE 7th International Conference on Industrial Engineering and Applications (ICIEA), Bangkok, Thailand, 16-21 April 2020; pp. 905-911.

35. Jing, C.; Destech Publications, I. Research on Location of Sales Logistics Network Nodes Based on Chaotic Optimization Algorithm. In Proceedings of the 3rd International Conference on Vehicle, Mechanical and Electrical Engineering (ICVMEE), Wuhan, China, 30-31 July 2016; pp. 71-75.

36. Sun, Y.; Geng, N.; Gong, S.; Yang, Y. Research on improved genetic algorithm in path optimization of aviation logistics distribution center. J. Intell. Fuzzy Syst. 2020, 38, 29-37. [CrossRef]

37. Hu, Y.; Zhang, K.; Yang, J.; Wu, Y. Application of Hierarchical Facility Location-Routing Problem with Optimization of an Underground Logistic System: A Case Study in China. Math. Probl. Eng. 2018, 2018. [CrossRef]

38. Deng, Y.; Zheng, Y.; Li, J. Route optimization model in collaborative logistics network for mixed transportation problem considered cost discount based on GATS. J. Ambient. Intell. Humaniz. Comput. 2019, 10, 409-416. [CrossRef]

39. Pinho, T.M.; Coelho, J.P.; Veiga, G.; Moreira, A.P.; Boaventura-Cunha, J. Soft computing optimization for the biomass supply chain operational planning. In Proceedings of the 13th APCA International Conference on Control and Soft Computing (CONTROLO), Ponta Delgada, Portugal, 4-6 June 2018; pp. 259-264.

40. Guo, J.; Fang, J.; Gen, M. Dynamic Joint Construction And Optimal Strategy Of Multi-Objective Multi Period Multi-Stage Reverse Logistics Network: A Case Study Of Lead Battery In Shanghai. In Proceedings of the 28th International Conference on Flexible Automation and Intelligent Manufacturing (FAIM)-Global Integration of Intelligent Manufacturing and Smart Industry for Good of Humanity, Columbus, OH, USA, 11-14 June 2018; pp. 1171-1178.

41. Yang, S.; Wang, J.; Deng, X. Research on node location of underground mining, dressing and backfilling system based on particle swarm optimization. J. Min. Saf. Eng. 2020, 37, 359-365. 
42. Cao, Y.; Liu, M.; Xing, Y.; Li, G.; Luo, J.; Gui, X. Current situation and prospect of underground coal preparation technology. J. Min. Saf. Eng. 2020, 37, 192-201.

43. Alexandridis, A.; Famelis, I.T.; Tsitouras, C. Particle Swarm Optimization for Complex Nonlinear Optimization Problems. In Proceedings of the International Conference On Numerical Analysis And Applied Mathematics 2015 (ICNAAM-2015), Rhodes, Greece, 23-29 September 2015.

44. Xu, M.; Zhang, W.; Qu, R.; Wang, J. An Improved Partial Swarm Optimization Algorithm for Solving Nonlinear Equation Problems. In Proceedings of the 2014 11th World Congress On Intelligent Control And Automation (WCICA), Shenyang, China, 29 June-4 July 2014; pp. 3600-3604.

45. Mo, Y.; Liu, H.; Wang, Q. Conjugate direction particle swarm optimization solving systems of nonlinear equations. Comput. Math. Appl. 2009, 57, 1877-1882. [CrossRef]

46. Sahu, D.R.; Agarwal, R.P.; Singh, V.K. A Third Order Newton-Like Method and Its Applications. Mathematics $2019,7,31$. [CrossRef]

47. Lai, K.K.; Mishra, S.K.; Ram, B. On q-Quasi-Newton's Method for Unconstrained Multiobjective Optimization Problems. Mathematics 2020, 8, 616. [CrossRef]

48. Ezquerro, J.A.; Hernandez-Veron, M.A. The Newtonian Operator and Global Convergence Balls for Newton's Method. Mathematics 2020, 8, 1074. [CrossRef]

49. Wang, X.; Tao, Y. A New Newton Method with Memory for Solving Nonlinear Equations. Mathematics 2020, 8, 108. [CrossRef]

50. Antonio Ezquerro, J.; Angel Hernandez-Veron, M. How to Obtain Global Convergence Domains via Newton's Method for Nonlinear Integral Equations. Mathematics 2019, 7, 553. [CrossRef] 